\title{
Lepton masses in a supersymmetric 3-3-1 model
}

\author{
J. C. Montero, ${ }^{*}$ V. Pleitez, ${ }^{\dagger}$ and M. C. Rodriguez \\ Instituto de Física Teórica, Universidade Estadual Paulista, Rua Pamplona, 145, 01405-900 São Paulo, SP, Brazil
}

(Received 19 December 2001; published 15 May 2002)

\begin{abstract}
We consider the mass generation for both charginos and neutralinos in a 3-3-1 supersymmetric model. We show that $R$-parity breaking interactions leave the electron and one of the neutrinos massless at the tree level. However, the same interactions induce masses for these particles at the 1-loop level. Unlike the similar situation in the minimal supersymmetric standard model, the masses of the neutralinos are related to the masses of the charginos.
\end{abstract}

DOI: 10.1103/PhysRevD.65.095008

PACS number(s): 12.60.Jv, 14.60.Pq

\section{INTRODUCTION}

The generation of neutrino masses is an important issue in any realistic extension of the standard model. In general, the values of these masses (of the order of, or less than, $1 \mathrm{eV}$ ) that are needed to explain all neutrino oscillation data [1-3] are not enough to put strong constraints on model building. This means that several models can induce neutrino masses and mixing compatible with experimental data. So, instead of proposing models built just to explain neutrino properties, it is more useful to consider what are the neutrino masses that are predicted in any particular model which has a motivation other than the explanation of neutrino physics. For instance, the 3-3-1 model was proposed as a possible symmetry on the lightest lepton sector $\left(\nu_{e}, e^{-}, e^{+}\right)_{L}$ [4]. Once that symmetry is assumed it has to be implemented in the rest of the leptons and also in the quark sector. Like in the standard model, if we do not introduce right-handed neutrinos and/or violation of the total lepton number, the neutrinos remain massless at any order in perturbation theory. In this vein some effort has been done to produce neutrino masses in the context of that 3-3-1 model and some of its extensions [5].

In this work we consider the generation of neutrino masses in a supersymmetric 3-3-1 model with broken $R$ parity. We show that, as an effect of the mixing among all leptons of the same charge, at the tree level only one charged lepton and one neutrino remain massless but they gain mass through radiative corrections. In order to compare this model we do the same calculations in the context of the minimal supersymmetric standard model with $R$ broken parity also. In both cases we are not assuming that sneutrinos gain nonvanishing vacuum expectation values (VEVs); i.e., the only nonzero VEVs are those of the scalars of the nonsupersymmetric models.

The outline of this work is as follows. In Sec. II we review the origin of the lepton masses in the minimal supersymmetric standard model context under the same assumptions that we will use in the case of the 3-3-1 supersymmetric

\footnotetext{
*Email address: montero@ift.unesp.br

†Email address: vicente@ift.unesp.br

‡Email address: mcr@ift.unesp.br
}

model. In Sec. III we consider the supersymmetric version of a 3-3-1 model which has only three triplets of Higgs scalars. We explicitly show that leptons gain mass only as a consequence of their mixing with gauginos and Higgsinos. Our conclusions are found in the last section.

\section{NEUTRINO MASSES IN THE MSSM}

Let us consider in this section the lepton masses in the minimal supersymmetric standard model (MSSM) [6]. In this model the interactions are written in terms of the lefthanded (right-handed) $\hat{L} \sim(\mathbf{2},-1)\left[\hat{l}^{c} \sim(\mathbf{1}, 2)\right]$ leptons, lefthanded (right-handed) quarks $\hat{Q} \sim(\mathbf{2}, 1 / 3) \quad\left[\hat{u}^{c} \sim(\mathbf{1}\right.$, $\left.-4 / 3), \hat{d}^{c} \sim(\mathbf{1}, 2 / 3)\right]$; and the Higgs doublets $\hat{H}_{1} \sim(\mathbf{2}$, -1), $\hat{H}_{2} \sim(\mathbf{2}, 1)$. With those multiplets the superpotential that conserves $R$-parity is given by $W_{2 R C}+W_{3 R C}+\bar{W}_{2 R C}$ $+\bar{W}_{3 R C}$, where

$$
W_{2 R C}=\mu \epsilon \hat{H}_{1} \hat{H}_{2} \text {, }
$$

$$
W_{3 R C}=\epsilon \hat{L}_{a} f_{a b}^{l} \hat{H}_{1} \hat{l}_{b}^{c}+\epsilon \hat{Q}_{i} f_{i j}^{u} \hat{H}_{2} \hat{u}_{j}^{c}+\epsilon \hat{Q}_{i} f_{i j}^{d} \hat{H}_{1} \hat{d}_{j}^{c},
$$

while the $R$-parity violating terms are given by $W_{2 R V}$ $+W_{3 R V}+\bar{W}_{2 R V}+\bar{W}_{3 R V}$, where

$$
\begin{aligned}
& W_{2 R V}=\mu_{0 a} \epsilon \hat{L}_{a} \hat{H}_{2}, \\
& W_{3 R V}=\epsilon \hat{L}_{a} \lambda_{a b c} \hat{L}_{b} \hat{l}_{c}^{c}+\epsilon \hat{L}_{a} \lambda_{a i j}^{\prime} \hat{Q}_{i} \hat{d}_{j}^{c}+\hat{u}_{i}^{c} \lambda_{i j k}^{\prime \prime} \hat{d}_{j}^{c} \hat{d}_{k}^{c},
\end{aligned}
$$

and we have suppressed $S U(2)$ indices; $\epsilon$ is the antisymmetric $S U(2)$ tensor. Above, and below in the following, the subindices $a, b, c$ run over the lepton generations $e, \mu, \tau$ but a superscript $c$ indicates charge conjugation; $i, j, k=1,2,3$ denote quark generations. We also have to add the soft terms that break the supersymmetry: 


$$
\begin{aligned}
\mathcal{L}_{\text {soft }}= & -\frac{1}{2}\left(\sum_{p=1}^{3} m_{\lambda} \lambda_{A}^{p} \lambda_{A}^{p}+m^{\prime} \lambda_{B} \lambda_{B}+\text { H.c. }\right)-M_{L}^{2} \widetilde{L}^{\dagger} \widetilde{L} \\
& -M_{l}^{2} \widetilde{l^{\dagger}} \widetilde{l}^{c}-M_{Q}^{2} \widetilde{Q}^{\dagger} \widetilde{Q}-M_{u}^{2} \widetilde{u^{\dagger \dagger}} \widetilde{u}^{c}-M_{d}^{2} \widetilde{d^{c \dagger}} \widetilde{d}^{c} \\
& -M_{1}^{2} \widetilde{H}_{1}^{\dagger} \widetilde{H}_{1}-M_{2}^{2} \widetilde{H}_{2}^{\dagger} \widetilde{H}_{2}-\left[A_{L} H_{1} \widetilde{L} \widetilde{l}^{c}+A_{U} H_{2} \widetilde{Q} \widetilde{u}^{c}\right. \\
& +A_{D} H_{1} \widetilde{Q} \widetilde{d}^{c}+M_{12}^{2} H_{1} H_{2}+B H_{2} L+C_{1} \widetilde{L} \widetilde{L} \widetilde{l}^{c} \\
& \left.+C_{2} \widetilde{L} \widetilde{Q} \widetilde{d}^{c}+C_{3} \widetilde{u}^{c} \widetilde{d}^{c} \widetilde{d}^{c}+\text { H.c. }\right],
\end{aligned}
$$

where $p$ is an $S U(2)$ index and $\lambda_{A}, \lambda_{B}$ are the supersymmet- ric partners of the respective gauge vector bosons but we have omitted generation indices and the gluino-mass terms.

With the interactions in Eq. (1) it is possible to give mass to all charged fermions in the model (see below) but neutrinos remain massless. Hence, we must introduce $R$-parity violating terms like those in Eq. (2). Some of the coupling constants in that expression should be set to zero in order to avoid a too fast proton decay. Defining the basis $\Psi_{M S S M}^{0}$ $=\left(\nu_{e}, \nu_{\mu}, \nu_{\tau},-i \lambda_{A}^{3},-i \lambda_{B}, \widetilde{H}_{1}^{0}, \widetilde{H}_{2}^{0}\right)^{T}$, the mass term is $-(1 / 2)\left[\Psi_{M S S M}^{0 T} Y_{M S S M}^{0} \Psi^{0}+\right.$ H.c. $]$, where $Y_{M S S M}^{0}$ is the mass matrix

$$
Y_{M S S M}^{0}=\left(\begin{array}{ccccccc}
0 & 0 & 0 & 0 & 0 & 0 & -\mu_{0 e} \\
0 & 0 & 0 & 0 & 0 & 0 & -\mu_{0 \mu} \\
0 & 0 & 0 & 0 & 0 & 0 & -\mu_{0 \tau} \\
0 & 0 & 0 & m_{\lambda} & 0 & M_{Z} s_{\beta} c_{W} & -M_{Z} c_{\beta} c_{W} \\
0 & 0 & 0 & 0 & m^{\prime} & M_{Z} s_{\beta} s_{W} & -M_{Z} c_{\beta} s_{W} \\
0 & 0 & 0 & M_{Z} s_{\beta} c_{W} & M_{Z} s_{\beta} s_{W} & 0 & \mu \\
-\mu_{0 e} & -\mu_{0 \mu} & -\mu_{0 \tau} & -M_{Z} c_{\beta} c_{W} & -M_{Z} c_{\beta} s_{W} & \mu & 0
\end{array}\right),
$$

where $s_{\beta}=\sin \beta, s_{W}=\sin \theta_{W}$, etc. are defined as $\tan \beta$ $=v_{2} / v_{1}$ and $\theta_{W}$ is the weak mixing angle. The matrix in Eq. (4) is generated only by the two usual vacuum expectation values of the two scalars and by the $R$-parity breaking terms $\mu_{0 a}$. The mass matrix is similar to that in Refs. [7-9] but we have included the three neutrinos and we are neither assuming that sneutrinos gain nonzero vacuum expectation values nor introduce sterile neutrinos like in Ref. [10]. The mass matrix in Eq. (4) has two zero eigenvalues: it has determinant equal to zero and its secular equation which gives the eigenvalues, $x$, has the form $x^{2}$ times a polynomial of five degrees; thus there are two neutrinos $\nu_{1,2}$, which are massless at the tree level. Using $\tan \beta=1$ and $M_{Z}$ $=91.187 \mathrm{GeV}, \quad s_{W}^{2}=0.223, \quad \mu_{0 e}=\mu_{0 \mu}=0, \quad \mu_{0 \tau}$ $=10^{-4} \mathrm{GeV}$ (this value is consistent with that of Ref. [8]), $\mu=100 \mathrm{GeV}, m=250 \mathrm{GeV}, m^{\prime}=-200 \mathrm{GeV}$, we obtain besides the two massless neutrinos a massive one with $m_{\nu_{3}}$ $=-3 \times 10^{-3} \mathrm{eV}$, and four heavy neutralinos with masses 267.40,-199.99,-117.40, and 100.0 GeV. These zero eigenvalues are a product of the matrix structure in Eq. (4) and there is no symmetry to protect the neutrinos from gaining mass by radiative corrections. On the other hand, if $\mu_{0 a}$ $=0, a=e, \mu, \tau$, all neutrinos remain massless at the tree level. In this case it is the $R$-parity and total lepton number conservation that protect neutrinos of gain masses. The neutralino masses above are consistent with those of Ref. [8]: two states are massless and the other ones have masses of the order $O\left(M_{Z}\right)$. More realistic neutrino masses require radiative corrections $[9,11-13]$. Here we will only consider the neutrino masses generated by radiative corrections arisen from the interactions given in Eqs. (1) and (2) and only two VEVs. We have in this case the interactions

$$
\begin{gathered}
-\frac{\lambda_{a b c}}{3}\left(\bar{\nu}_{a L} l_{b R} \widetilde{l}_{c}+\bar{\nu}_{a R}^{c} l_{b L}^{c} \widetilde{l}_{c}^{*}\right), \\
-\frac{\lambda_{a i j}^{\prime}}{3}\left(\bar{\nu}_{a L} d_{i R} \widetilde{d}_{j}+\bar{\nu}_{a R}^{c} d_{i L}^{c} \widetilde{d}_{j}^{*}\right)+\text { H.c. },
\end{gathered}
$$

and the 1-loop diagrams like those in Ref. [7] arise. Notice however that if we introduce a discrete symmetry (called $Z_{3}^{\prime}$ later on), $\hat{L}_{e, \mu} \rightarrow-\hat{L}_{e, \mu}$, and all other fields are even under this transformation, we have that

$$
\begin{aligned}
& \mu_{0 e}=\mu_{0 \mu}=0 ; \\
& \lambda_{e b c}=0,(b, c=\mu, \tau) ; \\
& \lambda_{\mu b c}=0, \quad(b, c=e, \tau) ; \\
& \lambda_{e i j}^{\prime}=\lambda_{\mu i j}^{\prime}=0, \quad(i, j=1,2,3) ;
\end{aligned}
$$

and the $\nu_{e}, \nu_{\mu}$ neutrinos will remain massless at all order in perturbation theory. It is also possible to choose the symmetry such as $L_{e, \tau} \rightarrow-L_{e, \tau}$ while all other fields remain invariant. In this case we have that $\nu_{e}$ and $\nu_{\tau}$ remain massless. However, if no discrete symmetry is imposed neutrinos gain mass through a 1-loop effect like in Ref. [7]. 
Next, let us consider the charged sector. With the interactions in Eq. (1) it is possible to give mass to all charged fermions. Denoting

$$
\begin{aligned}
& \phi_{M S S M}^{+}=\left(e^{c}, \mu^{c}, \tau^{c},-i \lambda_{W}^{+}, \widetilde{H}_{2}^{+}\right)^{T}, \\
& \phi_{M S S M}^{-}=\left(e, \mu, \tau,-i \lambda_{W}^{-}, \widetilde{H}_{1}^{-}\right)^{T},
\end{aligned}
$$

where all the fermionic fields are still Weyl spinors, we can

$$
X_{M S S M}=\left(\begin{array}{cc}
-f_{e e}^{l} v_{1} & -f_{e \mu}^{l} v_{1} \\
-f_{e \mu}^{l} v_{1} & -f_{\mu \mu}^{l} v_{1} \\
-f_{e \tau}^{l} v_{1} & -f_{\mu \tau}^{l} v_{1} \\
0 & 0 \\
\mu_{0 e} & \mu_{0 \mu}
\end{array}\right.
$$

With $f_{e e}^{l}=2.7 \times 10^{-4}, \quad f_{\mu \mu}^{l}=3.9 \times 10^{-3}, \quad f_{\tau \tau}^{l}=1.6 \times 10^{-2}$, $f_{e \mu}^{l}=f_{e \tau}^{l}=f_{\mu \tau}^{l}=10^{-7}$ we obtain from Eq. (9) the masses $0.0005,0.105,1.777$ (in $\mathrm{GeV}$ ) for the usual leptons, and 4.3 and $81 \mathrm{TeV}$ for the charginos. We see by comparing Eq. (4) with Eq. (9) that there is no relation between the charged lepton masses and the neutralino masses. Notice also that all charged leptons gain masses at the tree level. We will not consider this model (or some of its extensions) further since it has been well studied in literature [7-9,11-13].

\section{A SUPERSYMMETRIC 3-3-1 MODEL}

In the nonsupersymmetric 3-3-1 model [4] the fermionic representation content is as follows: left-handed leptons $L$ $=\left(\nu_{a}, l_{a}, l_{a}^{c}\right)_{L} \sim(\mathbf{1}, 3,0), a=e, \mu, \tau$; left-handed quarks $Q_{1 L}$ $=\left(u_{1}, d_{1}, J\right) \sim(3,3,2 / 3), Q_{\alpha L}=\left(d_{\alpha}, u_{\alpha}, j_{\beta}\right) \sim\left(3,3^{*},-1 / 3\right)$, $\alpha=2,3, \quad \beta=1,2$; and in the right-handed components we have $u_{i}^{c}, d_{i}^{c}, i=1,2,3$, that transform as in the SM, and the exotic quarks $J^{c} \sim\left(3^{*}, \mathbf{1},-5 / 3\right), j_{\beta} \sim\left(3^{*}, \mathbf{1}, 4 / 3\right)$. The minimal scalar representation content is formed by three scalar triplets: $\quad \eta \sim(\mathbf{1}, \mathbf{3}, 0)=\left(\eta^{0}, \eta_{1}^{-}, \eta_{2}^{+}\right)^{T} ; \quad \rho \sim(\mathbf{1}, \mathbf{3},+1)$ $=\left(\rho^{+}, \rho^{0}, \rho^{++}\right)^{T}$ and $\chi \sim(\mathbf{1}, \mathbf{3},-1)=\left(\chi^{-}, \chi^{--}, \chi^{0}\right)^{T}$, and one scalar sextet $S \sim(\mathbf{1}, \mathbf{6}, 0)$. We can avoid the introduction of the sextet by adding a charged lepton transforming as a singlet $[14,15]$. Notwithstanding, here we will omit both the sextet and the exotic lepton. A seesaw-type mechanism will be implemented by the mixing with supersymmetric partners, Higgsinos or gauginos. The complete set of fields in the 3-3-1 supersymmetric model has been given in Refs. [16,17]. We will denote, as in the previous section, the respective superfields as $\hat{L}$ and so on. We recall that in the nonsupersymmetric 3-3-1 model with only the three triplets the charged lepton masses are not yet the physical ones: $0, m$, $-m$.

We will show how in the present model supersymmetry the $R$-violating interactions give the correct masses to $e, \mu$ define $\Psi_{M S S M}^{ \pm}=\left(\phi_{M S S M}^{+}, \phi_{M S S M}^{-}\right)^{T}$, and the mass term - (1/2) $\left[\Psi_{M S S M}^{ \pm T} Y_{M S S M}^{ \pm} \Psi_{M S S M}^{ \pm}+\right.$H.c. $]$where $Y^{ \pm}$is the mass matrix given by

$$
Y_{M S S M}^{ \pm}=\left(\begin{array}{cc}
0 & X_{M S S M}^{T} \\
X_{M S S M} & 0
\end{array}\right)
$$

with

$\left.\begin{array}{ccc}-f_{e \tau}^{l} v_{1} & 0 & 0 \\ -f_{\mu \tau}^{l} v_{1} & 0 & 0 \\ -f_{\tau \tau}^{l} v_{1} & 0 & 0 \\ 0 & m_{\lambda} & \sqrt{2} M_{W} c_{\beta} \\ \mu_{0 \tau} & \sqrt{2} M_{W} s_{\beta} & \mu\end{array}\right)$.

and $\tau$, even without a sextet or the charged lepton singlet. We have the Higgsinos $\tilde{\eta}, \tilde{\rho}, \tilde{\chi}$ and their respective primed fields which have the same charge assignment of the triplets $\eta, \rho$ and $\chi$, for details see Ref. [16].

Because of the fact that in the supersymmetric model we have the gauginos and Higgsinos (for details on the Lagrangian of the model see [16]), when the $R$ parity is broken we have in analogy with the MSSM, but with important differences, a mixture between the usual leptons and the gauginos and Higgsinos.

One part of the superpotential is given by $W_{2}+\bar{W}_{2}$ where

$$
W_{2}=\mu_{0 a} \hat{L}_{a} \hat{\eta}^{\prime}+\mu_{\eta} \hat{\eta} \hat{\eta}^{\prime}+\mu_{\rho} \hat{\rho} \hat{\rho}^{\prime}+\mu_{\chi} \hat{\chi} \hat{\chi}^{\prime}
$$

$a=e, \mu, \tau ;$ and $W_{3}+\bar{W}_{3}$ where

$$
\begin{aligned}
W_{3}= & \lambda_{1 a b c} \epsilon \hat{L}_{a} \hat{L}_{b} \hat{L}_{c}+\lambda_{2 a b} \epsilon \hat{L}_{a} \hat{L}_{b} \hat{\eta}+\lambda_{3 a} \hat{L}_{a} \hat{\rho} \hat{\chi}+f_{1} \epsilon \hat{\eta} \hat{\rho} \hat{\chi} \\
& +f_{1}^{\prime} \epsilon \hat{\eta}^{\prime} \hat{\rho}^{\prime} \hat{\chi}^{\prime}+\lambda_{\alpha a i}^{\prime} \hat{Q}_{\alpha} \hat{L}_{a} \hat{d}_{i}^{c}+\lambda_{i j k}^{\prime \prime} \hat{u}_{i}^{c} \hat{d}_{j}^{c} \hat{d}_{k}^{c}+\lambda_{i j \beta}^{\prime \prime \prime} \hat{u}_{i}^{c} \hat{u}_{j}^{c} \hat{j}_{\beta}^{c} \\
& +\lambda_{i \beta}^{\prime \prime \prime \prime} \hat{d}_{i}^{c} \hat{J}^{c} \hat{j}_{\beta}^{c}+\kappa_{1 i} \hat{Q}_{1} \hat{\eta}^{\prime} \hat{u}_{i}^{c}+\kappa_{2 i} \hat{Q}_{1} \hat{\rho}^{\prime} \hat{d}_{i}^{c}+\kappa_{3} \hat{Q}_{1} \hat{\chi}^{\prime} \hat{J}^{c} \\
& +\kappa_{4 \alpha i} \hat{Q}_{\alpha} \hat{\eta}^{c} \hat{d}_{i}^{c}+\kappa_{5 \alpha i} \hat{Q}_{\alpha} \hat{\rho} \hat{u}_{i}^{c}+\kappa_{6 \alpha \beta} \hat{Q}_{\alpha} \hat{\chi}_{\hat{j}}^{c},
\end{aligned}
$$

with $\epsilon$ the completely antisymmetric tensor of $S U(3)$ but we have omitted the respective indices; the generation indices are as follows: $a, b, c=e, \mu, \tau$ and $i, j, k=1,2,3$.

The gaugino masses come from the soft terms shown in Appendix A, Eq. (A4). The $\mu_{0}, \lambda_{1}, \lambda_{3}, \lambda^{\prime}, \lambda^{\prime \prime}, \lambda^{\prime \prime \prime}$ and $\lambda^{\prime \prime \prime \prime}$ terms break the $R$ parity defined in this model as $R=$ $(-1)^{3 \mathcal{F}+2 \mathrm{~S}}$ where $\mathcal{F}=B+L, B(L)$ is the baryon (total lepton) number; $S$ is the spin. The $\lambda_{2}$ term of the superpotential $W_{3}$ implies interactions like [see Eq. (43) below] $\bar{\nu}_{a L} \tilde{\eta}_{2 R}^{-} \widetilde{l}_{b}^{*}$ $-\bar{\nu}_{a L} \tilde{\eta}_{1 R}^{+} \widetilde{l}_{b}$ and we have also the interactions 


$$
\mathcal{L}_{\eta}=\int d^{4} \theta \hat{\bar{\eta}} e^{2 g \hat{V}} \hat{\eta},
$$

where $\hat{V}$ is the superfield related to the $V^{a}$ gauge boson of $S U(3)_{L}$. This interaction mixes Higgsinos with gauginos as shown in Ref. [17].

The parameters $\mu_{\eta}$ and $\mu_{\rho}$ are the equivalent of the $\mu$ parameter in the MSSM [6]. The terms proportional to $\lambda_{2}$ and $\mu_{\chi}$ have no equivalent in the MSSM. The $\lambda^{\prime}$ and $\lambda^{\prime \prime}$ coupling constants are constrained by the proton decay such that $[18]$

$$
\lambda_{11 j}^{\prime \prime} \lambda_{a 2 j}^{\prime}<10^{-24}
$$

assuming the superpartner masses in the range of $1 \mathrm{TeV}$.

\section{A. Charged lepton masses}

In this model there are interactions like

$$
\begin{aligned}
-\frac{\lambda_{3 a}}{3}\left[\omega\left(l_{a} \tilde{\rho}^{+}+\bar{l}_{a} \overline{\tilde{\rho}^{+}}\right)+u\left(l_{a}^{c} \tilde{\chi}^{-}+\bar{l}_{a}^{c} \overline{\tilde{\chi}^{-}}\right)\right] \\
-\frac{1}{2} \mu_{0 a}\left[l \tilde{\eta}_{1}^{\prime+}+\bar{l} \overline{\tilde{\eta}_{1}^{\prime+}}+l^{c}{\tilde{\eta_{2}^{\prime}}}^{\prime-}+\bar{l}^{c} \overline{\tilde{\eta}_{2}^{\prime-}}\right],
\end{aligned}
$$

which imply a general mixture in both neutral and charged sectors. Let us first consider the charged lepton masses. Denoting

$$
\begin{aligned}
& \phi^{+}=\left(e^{c}, \mu^{c}, \tau^{c},-i \lambda_{W}^{+},-i \lambda_{V}^{+}, \tilde{\eta}_{1}^{\prime+}, \tilde{\eta}_{2}^{+}, \tilde{\rho}^{+}, \tilde{\chi}^{\prime+}\right)^{T}, \\
& \phi^{-}=\left(e, \mu, \tau,-i \lambda_{W}^{-},-i \lambda_{V}^{-}, \tilde{\eta}_{1}^{-}, \tilde{\eta}_{2}^{\prime-}, \tilde{\rho}^{\prime-}, \tilde{\chi}^{-}\right)^{T},
\end{aligned}
$$

where all the fermionic fields are still Weyl spinors, we can also, as before, define $\Psi^{ \pm}=\left(\phi^{+} \phi^{-}\right)^{T}$, and the mass term $-(1 / 2)\left[\Psi^{ \pm T} Y^{ \pm} \Psi^{ \pm}+\right.$H.c. $]$where $Y^{ \pm}$is given by

$$
Y^{ \pm}=\left(\begin{array}{cc}
0 & X^{T} \\
X & 0
\end{array}\right),
$$

with

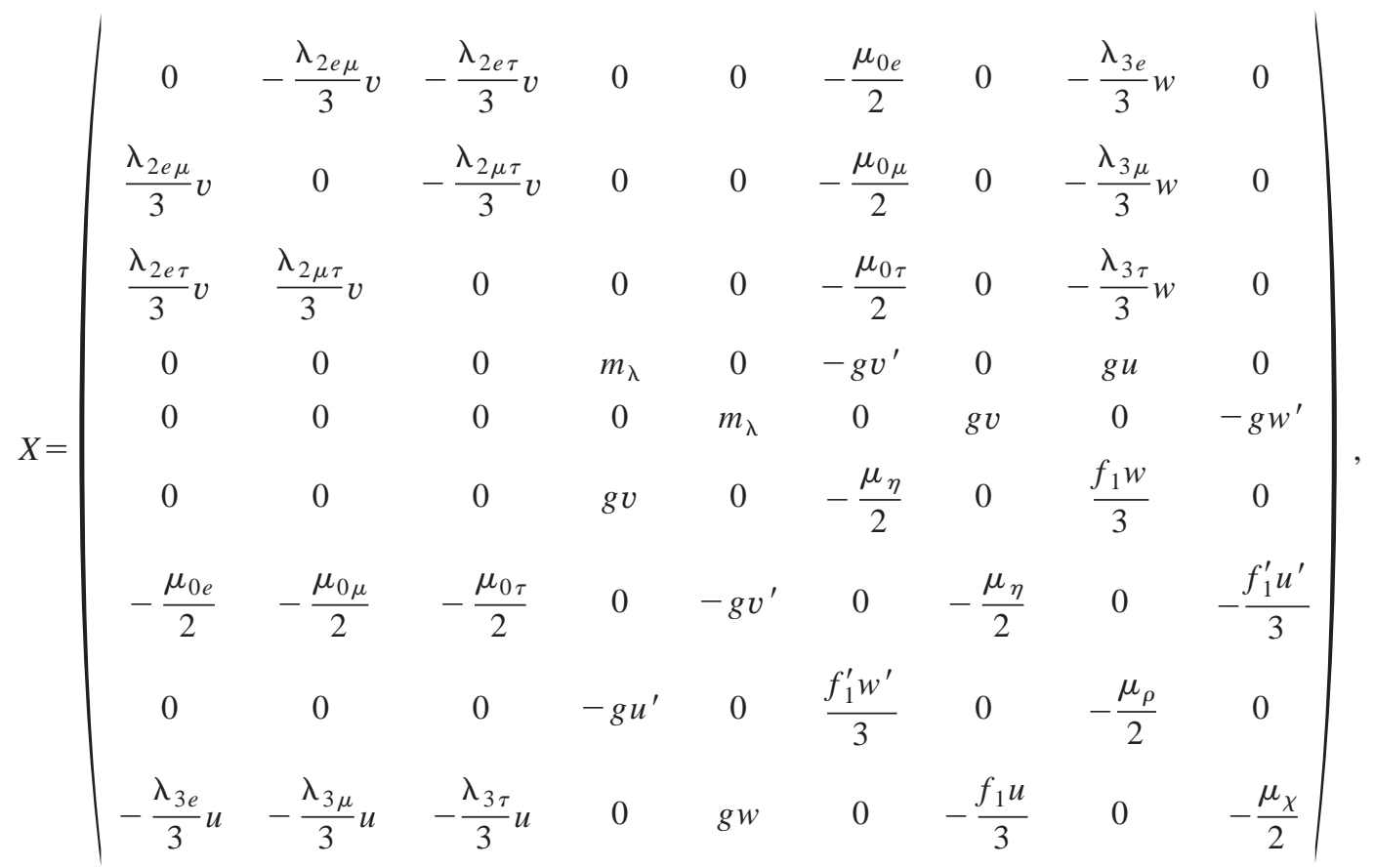

where we have defined

$$
\begin{gathered}
v=\frac{v_{\eta}}{\sqrt{2}}, \quad u=\frac{v_{\rho}}{\sqrt{2}}, \quad w=\frac{v_{\chi}}{\sqrt{2}}, \\
v^{\prime}=\frac{v_{\eta}^{\prime}}{\sqrt{2}}, \quad u^{\prime}=\frac{v_{\rho}^{\prime}}{\sqrt{2}}, \quad w^{\prime}=\frac{v_{\chi}^{\prime}}{\sqrt{2}} .
\end{gathered}
$$

The chargino mass matrix $Y^{ \pm}$is diagonalized using two unitary matrices, $D$ and $E$, defined by

$$
\tilde{\chi}_{i}^{+}=D_{i j} \Psi_{j}^{+}, \quad \tilde{\chi}_{i}^{-}=E_{i j} \Psi_{j}^{-}, i, j=1, \ldots, 9
$$

( $D$ and $E$ sometimes are denoted, in nonsupersymmetric theories, by $U_{R}^{l}$ and $U_{L}^{l}$, respectively). Then we can write the diagonal mass matrix as

$$
M_{S C M}=E^{*} X D^{-1}
$$




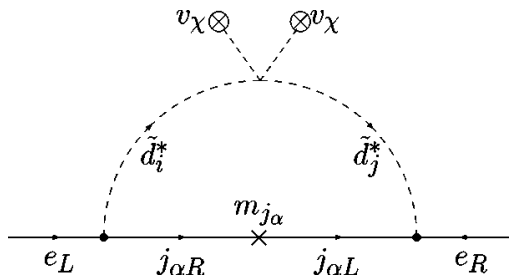

FIG. 1. Diagram generating the electron mass. There is also a contribution with $v_{\chi} \rightarrow v_{\chi^{\prime}}$. The left- and right-side vertices are proportional to $\lambda_{\alpha e i}^{\prime} / 3$ and $\lambda_{\alpha^{\prime} e j}^{\prime} / 3$, respectively.

To determine $E$ and $D$, we note that

$$
M_{S C M}^{2}=D X^{T} \cdot X D^{-1}=E^{*} X \cdot X^{T}\left(E^{*}\right)^{-1},
$$

and define the following Dirac spinors:

$$
\Psi\left(\tilde{\chi}_{i}^{+}\right)=\left(\tilde{\chi}_{i}^{+} \overline{\widetilde{\chi}}_{i}^{-}\right)^{T}, \quad \Psi^{c}\left(\tilde{\chi}_{i}^{-}\right)=\left(\tilde{\chi}_{i}^{-} \overline{\tilde{\chi}}_{i}^{+}\right)^{T},
$$

where $\tilde{\chi}_{i}^{+}$is the particle and $\tilde{\chi}_{i}^{-}$is the antiparticle $[6,17]$.

We have obtained the following masses (in $\mathrm{GeV}$ ) for the charged sector:

$$
3186.05,3001.12,584.85,282.30,204.55,149.41
$$

and the masses for the usual leptons (in GeV): $m_{e}=0, m_{\mu}$ $=0.1052$ and $m_{\tau}=1.777$. These values have been obtained by using the following values for the dimensionless parameters:

$$
\begin{aligned}
\lambda_{2 e \mu} & =0.001, \lambda_{2 e \tau}=0.001, \lambda_{2 \mu \tau}=0.393 \\
\lambda_{3 e} & =0.0001, \lambda_{3 \mu}=1.0, \lambda_{3 \tau}=1.0 \\
f_{1} & =0.254, f_{1}^{\prime}=1.0
\end{aligned}
$$

and for the mass dimension parameters (in $\mathrm{GeV}$ ) we have used

$$
\begin{aligned}
& \mu_{0 e}=\mu_{0 \mu}=0.0, \quad \mu_{0 \tau}=10^{-6}, \\
& \mu_{\eta}=300, \quad \mu_{\rho}=500, \mu_{\chi}=700, m_{\lambda}=3000 .
\end{aligned}
$$

We also use the constraint $V_{\eta}^{2}+V_{\rho}^{2}=(246 \mathrm{GeV})^{2}$ coming from $M_{W}$, where we have defined $V_{\eta}^{2}=v_{\eta}^{2}+v_{\eta}^{\prime 2}$ and $V_{\rho}^{2}$ $=v_{\rho}^{2}+v_{\rho}^{\prime 2}$. Assuming that $v_{\eta}=20 \mathrm{GeV}, v_{\eta}^{\prime}=v_{\rho}^{\prime}=1 \mathrm{GeV}$, and $2 v_{\chi}=v_{\chi}^{\prime}=2 \mathrm{TeV}$, the value of $v_{\rho}$ is fixed by the constraint above.

Notice that the electron is massless at the tree level. This is again a result of the structure of the mass matrix in Eq. (9) and there is not a symmetry that protects the electron to get a mass by loop corrections. Hence, it can gain mass through radiative corrections like that shown in Fig. 1. The interactions of the leptons with the sleptons written in terms of Dirac fermions (although we are using the same notation) are given by (and the respective Hermitian conjugate)

$$
\begin{aligned}
\mathcal{L}^{l}= & \frac{\lambda_{2 a b}}{3}\left[\overline{\tilde{\eta}_{2 R}^{-}}\left(l_{b L} \tilde{\nu}_{l a}-l_{a L} \tilde{\nu}_{l b}\right)+\overline{\tilde{\eta}_{R}^{0}}\left[\left(l_{a L} \widetilde{l}_{b}^{*}-l_{b L} \widetilde{l}_{a}^{*}\right)\right.\right. \\
& \left.\left.+\left(l_{b L}^{c} \tilde{l}_{a}-l_{a L}^{c} \tilde{l}_{b}\right)\right]+\overline{\tilde{\eta}_{1 R}^{+}}\left(l_{a L}^{c} \tilde{\nu}_{b L}-l_{b L}^{c} \tilde{\nu}_{a L}\right)\right] \\
& +\frac{\lambda_{3 a}}{3}\left[-\overline{\tilde{\rho}_{R}^{-}-} l_{a L} \chi^{-}+\overline{\tilde{\rho}}_{R}^{-}\left(l_{a L} \chi^{0}-l_{a L}^{c} \chi^{--}\right)\right. \\
& -\overline{\tilde{\chi}_{R}^{+}}\left(l_{a L} \rho^{++}-l_{a l}^{c} \rho^{0}\right)-\overline{\tilde{\chi}_{R}^{++}} l_{a L}^{c} \rho^{+}+\overline{\tilde{\rho}_{R}^{0}} l_{a L}^{c} \chi^{-} \\
& \left.+\overline{\tilde{\chi}_{R}^{0}} l_{a L} \rho^{+}\right]+\frac{\lambda_{\alpha a i}^{\prime}}{2}\left[\left(\bar{u}_{\alpha R} l_{a L}+\bar{j}_{\alpha R} l_{a L}^{c}\right) \tilde{d}_{i}^{*}\right. \\
& \left.+\bar{d}_{i R}^{c}\left(l_{a L} \tilde{u}_{\alpha}+l_{a L}^{c} \tilde{j}_{\alpha}\right)\right] .
\end{aligned}
$$

The $\lambda^{\prime}$ interactions generate the low vertices in Fig. 1. On the other hand, the interactions between the squarks, sleptons and scalars, see Appendix A, are given by the scalar potential. The soft part contributes only through the trilinear interactions

$$
V_{s o f t}=\epsilon_{1 a b}\left(\tilde{l}_{a} \tilde{l}_{b}^{*}-\tilde{l}_{a}^{*} \tilde{l}_{b}\right) \eta^{0}
$$

while the $D$ terms have only quartic interactions

$$
V_{D}=\frac{g^{2}}{4} \sum_{i}\left(X_{i}^{0} X_{i}^{0}+X_{a}^{\prime 0} X_{i}^{\prime 0}\right) \sum_{a}\left(\widetilde{l}_{a} \widetilde{l}_{a}^{*}+\frac{1}{2} \widetilde{\nu}_{a} \widetilde{\nu}_{a}^{*}\right)
$$

where $X_{i}^{0}=\chi^{0}, \eta^{0} ; X_{i}^{\prime 0}=\eta^{\prime 0}, \chi^{\prime 0} ;$ and $a=e, \mu, \tau$. From the $F$ terms we have contributions to both the trilinear interactions

$$
V_{3 F}=\left(\frac{\mu_{0 c} \lambda_{1 a b c}}{2}+\frac{\mu_{\chi} \lambda_{2 a b}}{6}\right)\left(\widetilde{l}_{a} \widetilde{l}_{b}^{*}-\widetilde{l}_{a}^{*} \widetilde{l}_{b}\right) \eta^{\prime 0}+\text { H.c. }
$$

and the quartic ones

$$
\begin{aligned}
V_{4 F}= & \left(\frac{\lambda_{3 d} \lambda_{1 d a b}}{3}+\frac{f_{1} \lambda_{2 a b}}{9}\right)\left(\tilde{l}_{a} \tilde{l}_{b}^{*}-\widetilde{l}_{a}^{*} \widetilde{l}_{b}\right) \rho^{0} \chi^{0} \\
& +\frac{4 \lambda_{2 a d} \lambda_{2 d b}}{9}\left(\tilde{l}_{a}^{*} \tilde{l}_{b}+\tilde{l}_{a} \tilde{l}_{b}^{*}\right) \eta^{0} \eta^{0} \\
& +\frac{\lambda_{3 a} \lambda_{3 b}}{9}\left(\widetilde{l}_{a} \tilde{l}_{b}^{*}+\tilde{\nu}_{a} \tilde{\nu}_{b}^{*}\right) \sum_{i} X_{i}^{0} X_{i}^{0},
\end{aligned}
$$

where $X_{i}^{0}=\chi^{0}, \rho^{0}$ which will contribute to the upper quartic vertex in Fig. 1. Due to the interactions given in Eqs. (25)(29), we can generate the appropriate mass to the electron. The dominant contributions, assuming the mass hierarchy $m_{\text {fermion }} \ll m_{\text {scalar }}$ where fermion means a fermion different from $j_{1,2}$ and scalar means $\tilde{\nu}, \tilde{l}, H$ ( $H$ denotes the heaviest Higgs scalar) and using the values of the masses and the parameters given in Eqs. (23), (24a) and (24d) we obtain that the dominant contribution to the electron mass is, up to logarithmic corrections, 


$$
m_{e} \propto \lambda_{\alpha e i}^{\prime} \lambda_{\alpha^{\prime} e j}^{\prime} V_{j}^{2} V_{b}^{2}\left(v_{\chi}^{2}+v_{\chi^{\prime}}^{2}\right) \frac{m_{j_{\alpha}}}{9 m_{\tilde{b}}^{2}},
$$

and with all the indices fixed, $V_{j}$ denotes mixing matrix elements in the two dimension $j_{1,2}$ space; $V_{b}$ means the same but in the $d$-like squark sector. We obtain $m_{e}$ $=0.0005 \mathrm{GeV}$ if $v_{\chi}$ and $v_{\chi}^{\prime}$ have the values already given above, and with $\lambda_{\alpha e i}^{\prime} \lambda_{\alpha^{\prime} e j}^{\prime} \approx 10^{-6}$, which imposes

$$
\frac{m_{j_{\beta}}}{m_{\tilde{b}}^{2}} \approx \frac{9 \times 10^{-4}}{V_{j}^{2} V_{b}^{2}} \mathrm{GeV}^{-1},
$$

or $m_{\tilde{b}}=33.33 \sqrt{m_{j_{2}}} V_{j} V_{b} \mathrm{GeV}$. Using $m_{j} \sim 250$ (320) $\mathrm{GeV}$ [19], we have $m_{\tilde{b}} \sim 526(596) V_{j} V_{b}$ GeV. With $V_{j} V_{b}$ $\sim 0.14(0.12)$ we obtain squarks masses of the order of $m_{\tilde{b}}$ $\sim 75 \mathrm{GeV}[20]$.

\section{B. Neutral lepton masses}

Like in the case of the charged sector, the neutral lepton masses are given by the mixing among neutrinos, induced by the $\mu_{0 a}$ term in Eq. (10), and the neutral Higgsinos and gauginos [17], and also by $\lambda_{2}$ and $\lambda_{3}$ in Eq. (11). The first two terms in Eq. (10) give the interactions between neutrinos and Higgsinos:

$$
\frac{1}{2} \mu_{0 a}\left[\nu_{a} \tilde{\eta}^{\prime 0}+\bar{\nu}_{a} \overline{\tilde{\eta}^{\prime 0}}\right]-\frac{\mu_{\eta}}{2}\left[\tilde{\eta}^{\prime 0} \tilde{\eta}^{0}+\overline{\tilde{\eta}^{\prime 0}} \overline{\tilde{\eta}^{0}}\right]
$$

and from Eq. (11) we have also the interactions

$$
\frac{\lambda_{3 a}}{3}\left[w\left(\nu_{a} \tilde{\rho}^{0}+\bar{\nu}_{a} \overline{\tilde{\rho}^{0}}\right)+u\left(\nu_{a} \tilde{\chi}^{0}+\bar{\nu}_{a} \overline{\tilde{\chi}^{0}}\right)\right] .
$$

These interactions imply a mass term for the neutrinos and neutralinos. The mass term in the basis

$$
\Psi^{0}=\left(\nu_{e} \nu_{\mu} \nu_{\tau}-i \lambda_{A}^{3}-i \lambda_{A}^{8}-i \lambda_{B} \tilde{\eta}^{0} \widetilde{\eta}^{\prime 0} \widetilde{\rho}^{0} \tilde{\rho}^{\prime 0} \tilde{\chi}^{0} \tilde{\chi}^{\prime 0}\right)^{T},
$$

is given by $-(1 / 2)\left[\left(\Psi^{0}\right)^{T} Y^{0} \Psi^{0}+\right.$ H.c. $]$ where

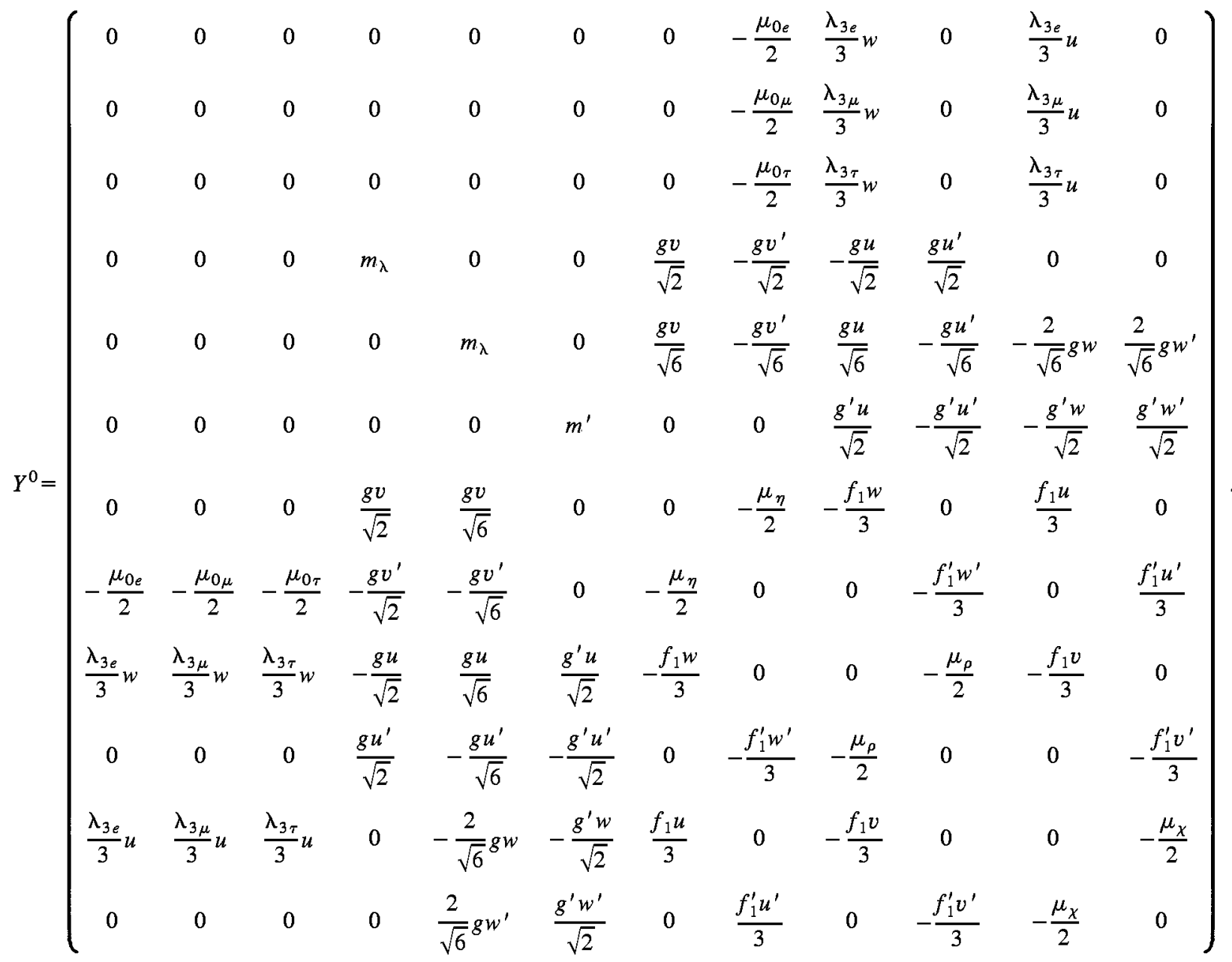


All parameters in Eq. (35), but $m^{\prime}$, are defined in Eqs. (18), (24a) and (24d); $g$ and $g^{\prime}$ denote the gauge coupling constant of $S U(3)_{L}$ and $U(1)_{N}$, respectively.

The neutralino mass matrix is diagonalized by a $12 \times 12$ rotation unitary matrix $N$, satisfying

$$
M_{N M D}=N^{*} Y^{0} N^{-1},
$$

and the mass eigenstates are

$$
\tilde{\chi}_{i}^{0}=N_{i j} \Psi_{j}^{0}, \quad j=1, \ldots, 12 .
$$

We can define the following Majorana spinor to represent the mass eigenstates:

$$
\Psi\left(\tilde{\chi}_{i}^{0}\right)=\left(\tilde{\chi}_{i}^{0} \overline{\tilde{\chi}}_{i}^{0}\right)^{T}
$$

As above the subindices $a, b, c$ run over the lepton generations $e, \mu, \tau$.

With the mass matrix in Eq. (35), at the tree level we obtain the eigenvalues (in $\mathrm{GeV}$ ),

$$
\begin{aligned}
& -4162.22,3260.48,3001.11,585.19,-585.19,453.22, \\
& -344.14,283.14,-272.0,
\end{aligned}
$$

and for the three neutrinos we obtain (in $\mathrm{eV}$ )

$$
m_{1}=0, m_{2} \approx-0.01, m_{3} \approx 1.44 \text {. }
$$

We have obtained the values in Eqs. (39) and (40) by choosing, besides the parameters in Eqs. (24a) and (24d), $m^{\prime}=$ $-3780.4159 \mathrm{GeV}$. Notice that the coupling constant $g^{\prime}$ and the parameter $m^{\prime}$ appear only in the mass matrix of the neutralinos; all the other parameters in Eq. (35) have already been fixed by the charged sector, see Eqs. (17), (24a) and (24d). The neutrino masses in Eq. (40) are of the order of magnitude for the Liquid Scintillation Neutrino Detector (LSND) and solar neutrino data. On the other hand, if we choose $m^{\prime}=-3780.4159 \mathrm{GeV}$ and $\mu_{0 \tau}=2 \times 10^{-8} \mathrm{GeV}$, we obtain (in $\mathrm{eV}$ )

$$
m_{1}=0.0, m_{2} \approx-5.47 \times 10^{-5}, m_{3} \approx 1.32 \times 10^{-2},
$$

which are of the order of magnitude required by the solar and atmospheric neutrino data. Notice also the sensibility of the neutrino masses in $\mu_{0 \tau}$ and $m^{\prime}$, and that if $\mu_{0 a}=0$, and $a$ $=e, \mu, \tau$, all neutrinos remain massless. The masses of the charged sector are insensible to the values of $\mu_{0 a}$ for all $a$ $=e, \mu, \tau$ which are suitable for generating the appropriate different neutrino mass spectra (see Appendix B).

We have obtained numerically the unitary matrices $E, D$ and $N$ which diagonalize the mass matrices in Eqs. (17) and (35) but we will not write them explicitly. The charged current is written in the mass-eigenstate basis as $\bar{l}_{L} \gamma^{\mu} V_{M N S} \nu_{L} W_{\mu}^{-}$with the Maki-Nakagawa-Sakata matrix [21] defined as $V_{M N S}=\mathcal{E}_{L}^{T} \mathcal{N}$, where $\mathcal{E}$ and $\mathcal{N}$ are the $3 \times 3$ submatrices of $E$ and $N$, respectively. Hence, we have

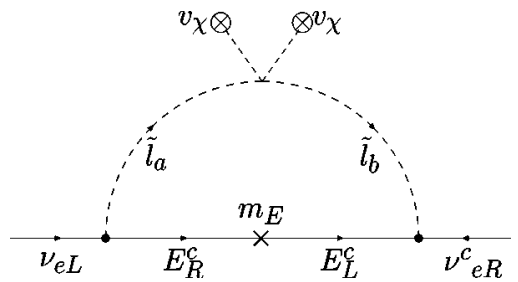

FIG. 2. Diagram generating the mass for the lightest neutrino. There is another dominant contribution with $v_{\chi} \rightarrow v_{\chi^{\prime}}$. Each vertex on the left and right side are proportional to $\lambda_{2 e} \tau / 3$ and $\lambda_{2 e \tau} / 3$, respectively.

$$
V_{M N S} \approx\left(\begin{array}{ccc}
1.000 & -0.004 & -0.001 \\
0.001 & -0.001 & 0.003 \\
-0.004 & -0.979 & -0.199
\end{array}\right)
$$

Notice that this leptonic mixing matrix is not orthogonal as it must be since we are omitting the mixture with the heavy charginos and neutralinos and one neutrino remains massless at the tree level. We can always rotate the neutral fields in such a way that the electron neutrino is the one which remains massless; or we can also assume $\mu_{0 e}=\lambda_{3 e}$ $=0$, so that the electron neutrino decouples from the other neutrinos and neutralinos. In this case, diagonal and nondiagonal mass terms in Eq. (35) will be induced by loop corrections like that in Fig. 2. Thus, a $3 \times 3$ nonorthogonal mixing matrix will appear in Eq. (42). Here we will only consider the order of magnitude of a mass generated by this process.

The massless neutrino can get a mass from the loop correction like that in Fig. 2 as a consequence of the Majorana mass term of the neutral lepton in the triplet. This is equivalent to the mechanism of Ref. [22] but now with a triplet of leptons instead of a neutral singlet. For instance, the $\lambda_{2}$ interactions will contribute in the left and right vertices in Fig. 2:

$$
\begin{aligned}
\mathcal{L}^{\nu}= & \frac{\lambda_{2 a b}}{3}\left[\overline{\tilde{\eta}_{2 R}^{-}}\left(\nu_{a L} \widetilde{l}_{b}-\nu_{b L} \widetilde{l}_{a}\right)+\overline{\tilde{\eta}_{1 R}^{+}}\left(\nu_{b L} \tilde{l}_{a}^{*}-\nu_{a L} \widetilde{l}_{b}^{*}\right)\right] \\
& +\frac{\lambda_{3 a}}{3}\left[\overline{\tilde{\chi}_{R}^{++}} \nu_{a L} \rho^{++}+\overline{\tilde{\rho}_{R}^{--}} \nu_{a L} \chi^{--}-\overline{\tilde{\chi}_{R}^{0}} \nu_{a L} \rho^{0}\right. \\
& \left.-\overline{\tilde{\rho}}_{R}^{0} \nu_{a L} \chi^{0}\right]+\frac{\lambda_{\alpha a i}^{\prime}}{3}\left[\bar{d}_{\alpha R} \nu_{a L} \widetilde{d}_{i}^{c}+\bar{d}_{i R}^{c} \nu_{a L} \widetilde{d}_{\alpha}\right]+\text { H.c. },
\end{aligned}
$$

these interactions generate the lower vertices of Fig. 2. The upper vertex are given in Eqs. (26)-(29). With these interactions we can generate the following small mass to the electron neutrino. In fact, assuming a hierarchy of the masses as in the last subsection, we obtain the dominant contribution to the $\nu_{e}$ mass, up to logarithmic corrections: 


$$
m_{\nu_{e}} \propto \lambda_{2 e a} \lambda_{2 e b} E_{e a} E_{e b} V_{\tilde{\tau}}^{2}\left(v_{\chi}^{2}+v_{\chi^{\prime}}^{2}\right) \frac{m_{a}}{9 m_{\tilde{\tau}}^{2}},
$$

where all the indices are fixed, $E_{e a}$ is the mixing matrix element defined in Eqs. (20) and (21), and $V_{\tilde{\tau}}$ denotes the mixing matrix element in the slepton sector. The charged lepton which gives the main contribution is the $\tau$ lepton: it has a large mass and the mixing angle is not too small, in fact $E_{e \tau}=0.004$. Since, $\lambda_{2 e \tau} \lambda_{2 e \tau} \sim 10^{-6}$ we obtain an electron neutrino mass of the order of $10^{-3} \mathrm{eV}$ if $m_{\tau} \approx 4$ $\times 10^{3} V_{\tilde{\tau}} \mathrm{GeV}$. If $V_{\tilde{\tau}}=0.02$ we have $m_{\tilde{\tau}} \sim 81 \mathrm{GeV}$ [20].

\section{CONCLUSIONS}

In the nonsupersymmetric 3-3-1 model [4] with only three scalar triplets $\eta, \rho \chi$ it is not possible to generate the observed charged lepton masses. Then, it is necessary to introduce a scalar sextet in order to get the appropriate masses. When we supersymmetrize the model and allow $R$-parity breaking interactions we can give to all known charged leptons and neutrinos the appropriate masses even without the introduction of a scalar sextet. Of course, in order to cancel anomalies we have to introduce another set of three triplets $\eta^{\prime}, \rho^{\prime} \chi^{\prime}$. In this case, although the correct values for the lepton masses can still be obtained, if the new VEVs $u^{\prime}, v^{\prime}$ and $w^{\prime}$ are zero, it was shown in Ref. [16] that in order to give mass to all the quarks in the model all these VEVs have to be different from zero. Hence, we have considered that the six neutral scalar components got a nonzero VEV.

As can be seen from Eq. (24), the charged lepton masses arise from a sort of seesaw mechanism since there are small mass parameters, as in Eq. $(24 \mathrm{c})$, related with $R$-parity breaking interactions, and large ones as in Eq. (24d), related with the mass scale of the supersymmetry breaking, this can be better appreciated in Eqs. (B1).

The same happens in the neutrino sector, see Eq. (B2). In a supersymmetric version of the model in which we add the sextet $(6,0)$, there is a fermionic non-Hermitian triplet under $S U(2) \otimes U(1)_{Y}$ that is part of a sextet under $S U(3)$ $\otimes U(1)_{N}$. This can also implement a seesaw mechanism for neutrino masses as was pointed out in Ref. [23]. The case of a Hermitian fermion triplet was considered in the context of the standard model in Refs. [24].

It is interesting to note that in the context of MSSM a $Z_{2}$ symmetry $[7,25]$,

$$
M \rightarrow-M, \quad V \rightarrow V, \quad X \rightarrow X
$$

where $M, V, X$ are matter, vector, and scalar superfields, respectively, forbids the $R$-parity breaking terms in Eq. (2). In the present model it happens the same way: the $R$-parity breaking terms in Eqs. (10) and (11) are forbidden. Notwithstanding the $Z_{3}$ symmetry [25],

$$
\begin{gathered}
\hat{L}, \hat{l}^{c} \rightarrow \hat{L}, \hat{l}^{c} ; \hat{H}_{1} \rightarrow \hat{H}_{1}, \hat{H}_{2} \rightarrow \hat{H}_{2} ; \\
\hat{Q} \rightarrow \omega \hat{Q}, \quad \hat{u}^{c} \rightarrow \omega^{-1} \hat{u}^{c}, \hat{d}^{c} \rightarrow \omega^{-1} \hat{d}^{c},
\end{gathered}
$$

where $\omega=e^{2 i \pi / 3}$, forbids the $B$ violating terms but allows the $L$ violating ones. This also happens in the present model. However, if we introduce an extra discrete $Z_{3}^{\prime}$ symmetry, such that $\hat{L}_{e} \rightarrow-\hat{L}_{e}$, with all other fields being even under this transformation, we have that $\mu_{0 e}=\lambda_{2 e a}=\lambda_{3 e}=\lambda_{\alpha e i}^{\prime}=0$, at all orders in perturbation theory. This does not modify the mass matrix in the charged sector in Eqs. (16) and (17), but forbids the electron neutrino to get a mass, at all orders in perturbation theory.

The present model will induce processes contributing to $\mu \rightarrow e \gamma, \tau \rightarrow e(\mu) \gamma,(g-2)_{\mu}$, and other exotic decays. However, these processes can be suppressed mainly by the scalar masses since these scalars do not enter explicitly in the mass matrix at the tree level. Some contributions to those processes are suppressed by the coupling constants themselves, like $\lambda_{2}$ 's in Eq. (24a); other ones which involve $\lambda_{3 \mu}, \lambda_{3 \tau}$ which are of the order unity can be suppressed by combining the mixing angles and masses of scalars or charginos sectors. A more detailed study of this issue will be done elsewhere [24].

In summary, we have analyzed the charged lepton and neutrino masses in an $R$-parity breaking supersymmetric 3-3-1 model. Unlike the MSSM model the electron and its neutrino remain massless at the tree level but gain masses at the one loop level. The resulting leptonic mixing matrix $V_{M N S}$ is nonorthogonal.

\section{ACKNOWLEDGMENTS}

This work was supported by Fundação de Amparoà Pesquisa do Estado de São Paulo (FAPESP), Conselho Nacional de Ciência e Tecnologia (CNPq) and by Programa de Apoio a Núcleos de Excelência (PRONEX).

\section{APPENDIX A: THE SCALAR POTENTIAL}

The interactions between the scalars of the theory are given by the scalar potential that is written as

$$
V_{331}=V_{D}+V_{F}+V_{\text {soft }},
$$

where the $V_{D}$ term is given by

$$
\begin{aligned}
V_{D}= & -\mathcal{L}_{D}=\frac{1}{2}\left(D^{a} D^{a}+D D\right) \\
= & \frac{g^{\prime 2}}{2}\left(\frac{2}{3} \widetilde{Q}_{1}^{\dagger} \widetilde{Q}_{1}-\frac{1}{3} \widetilde{Q}_{\alpha}^{\dagger} \widetilde{Q}_{\alpha}-\frac{2}{3} \widetilde{u}_{i}^{\dagger c} \widetilde{u}_{1}^{c}\right. \\
& \left.+\frac{1}{3} \widetilde{d}_{i}^{\dagger} \widetilde{d}_{1}^{c}+\rho^{\dagger} \rho-\chi^{\dagger} \chi-\rho^{\prime \dagger} \rho^{\prime}+\chi^{\prime \dagger} \chi^{\prime}\right)^{2} \\
& +\frac{g^{2}}{8} \sum_{i, j}\left(\widetilde{L}_{i}^{\dagger} \lambda_{i j}^{a} \widetilde{L}_{j}+\widetilde{Q}_{1 i}^{\dagger} \lambda_{i j}^{a} \widetilde{Q}_{1 j}\right. \\
& +\eta_{i}^{\dagger} \lambda_{i j}^{a} \eta_{j}+\rho_{i}^{\dagger} \lambda_{i j}^{a} \rho_{j}+\chi_{i}^{\dagger} \lambda_{i j}^{a} \chi_{j} \\
& -\widetilde{Q}_{\alpha i}^{\dagger} \lambda_{i j}^{* a} \widetilde{Q}_{\alpha j}-\eta_{i}^{\prime \dagger} \lambda_{i j}^{* a} \eta_{j}^{\prime}-\rho_{i}^{\prime \dagger} \lambda_{i j}^{* a} \rho_{j}^{\prime}-\chi_{i}^{\prime \dagger} \lambda_{i j}^{* a} \chi_{j}^{\prime}
\end{aligned}
$$

and the $F$ term is 


$$
\begin{aligned}
V_{F}= & -\mathcal{L}_{F}=\sum_{m} F_{m}^{*} F_{m} \\
= & \sum_{i, j, k}\left[\left|\frac{\mu_{0}}{2} \eta_{i}^{\prime}+\lambda_{1} \epsilon_{i j k} \widetilde{L}_{j} \widetilde{L}_{k}+\frac{2 \lambda_{2}}{3} \epsilon_{i j k} \eta_{j} \widetilde{L}_{k}+\frac{\lambda_{3}}{3} \epsilon_{i j k} \chi_{j} \rho_{k}\right|^{2}+\left|\frac{\mu_{\eta}}{2} \eta_{i}^{\prime}+\frac{\lambda_{2}}{3} \epsilon_{i j k} \widetilde{L}_{j} \widetilde{L}_{k}+\frac{f_{1}}{3} \epsilon_{i j k} \rho_{j} \chi_{k}+\frac{\kappa_{4 \alpha i j}}{3} \widetilde{Q}_{\alpha} \widetilde{d}_{j}^{c}\right|^{2}\right. \\
& +\left|\frac{\mu_{\rho}}{2} \rho_{i}^{\prime}+\frac{f_{1}}{3} \epsilon_{i j k} \chi_{j} \eta_{k}+\frac{\kappa_{5 \alpha i j}}{3} \widetilde{Q}_{\alpha} \widetilde{u}_{j}^{c}+\frac{\lambda_{3}}{3} \epsilon_{i j k} \widetilde{L}_{j} \chi_{k}\right|^{2}+\left|\frac{\mu_{\chi}}{2} \chi_{i}^{\prime}+\frac{f_{1}}{3} \epsilon_{i j k} \rho_{j} \eta_{k}+\frac{\kappa_{6 \alpha i \beta}}{3} \widetilde{Q}_{\alpha} \widetilde{j}_{\beta}^{c}+\frac{\lambda_{3}}{3} \epsilon_{i j k} \widetilde{L}_{j} \rho_{k}\right|^{2} \\
& +\left|\frac{\mu_{\eta}}{2} \eta_{i}+\frac{f_{1}^{\prime}}{3} \epsilon_{i j k} \rho_{j}^{\prime} \chi_{k}^{\prime}+\frac{\kappa_{1 i j}}{3} \widetilde{Q}_{1} \widetilde{u}_{j}^{c}\right|^{2}+\left|\frac{\mu_{\rho}}{2} \rho_{i}+\frac{f_{1}^{\prime}}{3} \epsilon_{i j k} \chi_{j}^{\prime} \eta_{k}^{\prime}+\frac{\kappa_{2 i j}}{3} \widetilde{Q}_{1} \widetilde{d}_{j}^{c}\right|^{2}+\left|\frac{\mu_{\chi}}{2} \chi_{i}+\frac{f_{1}^{\prime}}{3} \epsilon_{i j k} \rho_{j}^{\prime} \eta_{k}^{\prime}+\frac{\kappa_{3 i}}{3} \widetilde{Q}_{1} \widetilde{J}^{c}\right|^{2} \\
& +\left|\frac{\kappa_{1 i j}}{3} \eta_{i}^{\prime} \widetilde{u}_{j}^{c}+\frac{\kappa_{2 i j}}{3} \rho_{i}^{\prime} \widetilde{d}_{j}^{c}+\frac{\kappa_{3 i}}{3} \chi_{i}^{\prime} \widetilde{J}^{c}\right|^{2}+\left|\frac{\kappa_{4 \alpha i j}}{3} \eta_{i} \widetilde{d}_{j}^{c}+\frac{\kappa_{5 \alpha i j}}{3} \rho_{i} \widetilde{u}_{j}^{c}+\frac{\kappa_{6 \alpha i \beta}}{3} \chi_{i j} \widetilde{j}_{\beta}^{c}+\frac{\lambda_{\alpha i j}^{\prime}}{3} \widetilde{L}_{i} \widetilde{d}_{j}^{c}\right|^{2} \\
& +\left|\frac{\kappa_{1 i j}}{3} \widetilde{Q}_{1} \eta_{i}^{\prime}+\frac{\kappa_{5 \alpha i j}}{3} \widetilde{Q}_{\alpha} \rho_{i}^{\prime}+\frac{\lambda_{i j k}^{\prime \prime}}{3} \widetilde{d}_{i}^{c} \widetilde{d}_{k}^{c}+\frac{\lambda_{i j \beta}^{\prime \prime \prime}}{3} \widetilde{u}_{i}^{c} \widetilde{j}_{\beta}^{c}\right|^{2} \\
& \left.+\left|\frac{\kappa_{2 i j}}{3} \widetilde{Q}_{1} \rho_{i}^{\prime}+\frac{\kappa_{4 \alpha i j}}{3} \widetilde{Q}_{\alpha} \eta_{i}+\frac{\lambda_{\alpha i j}^{\prime}}{3} \widetilde{Q}_{\alpha} \widetilde{L}_{i}+\frac{2 \lambda_{i j k}^{\prime \prime}}{3} \widetilde{d}_{i}^{c} \widetilde{u}_{k}^{c}+\frac{\lambda_{j \beta}^{\prime \prime \prime}}{3} \widetilde{J}^{c} \widetilde{j}_{\beta}^{c}\right|^{2}\right] .
\end{aligned}
$$

Finally, the soft term is (the following soft terms do not include the exotic quarks)

$$
\begin{aligned}
V_{\text {soft }}= & -\mathcal{L}_{\text {soft }} \\
= & \frac{1}{2}\left(m_{\lambda} \sum_{w=1}^{8} \lambda_{A}^{w} \lambda_{A}^{w}+m^{\prime} \lambda_{B} \lambda_{B}+\text { H.c. }\right)+m_{L^{2}}^{2} \widetilde{L}^{\dagger} \widetilde{L}+m_{Q_{1}}^{2} \widetilde{Q}_{1}^{\dagger} \widetilde{Q}_{1}+\sum_{\alpha=2}^{3} m_{Q_{\alpha}}^{2} \widetilde{Q}_{\alpha}^{\dagger} \widetilde{Q}_{\alpha}+\sum_{i=1}^{3} m_{u_{i}}^{2} \widetilde{u}_{i}^{c \dagger} \tilde{u}_{i}^{c} \\
& +\sum_{i=1}^{3} m_{d_{i}}^{2} \widetilde{d}_{i}^{c \dagger} \widetilde{d}_{i}^{c}+m_{\eta}^{2} \eta^{\dagger} \eta+m_{\rho}^{2} \rho^{\dagger} \rho+m_{\chi}^{2} \chi^{\dagger} \chi^{\prime}+m_{\eta^{\prime}}^{2} \eta^{\prime \dagger} \eta^{\prime}+m_{\rho^{\prime}}^{2} \rho^{\prime \dagger} \rho^{\prime}+m_{\chi^{\prime}}^{2} \chi^{\prime \dagger} \chi^{\prime} \\
& +\left[M^{2} \sum_{i=1}^{3} \widetilde{L}_{i} \eta_{i}^{\dagger}+\varepsilon_{0} \sum_{i=1}^{3} \sum_{j=1}^{3} \sum_{k=1}^{3} \epsilon_{i j k} \widetilde{L}_{i} \widetilde{L}_{j} \widetilde{L}_{k}+\varepsilon_{1} \sum_{i=1}^{3} \sum_{j=1}^{3} \epsilon_{i j k} \widetilde{L}_{i} \widetilde{L}_{j} \eta_{k}+\varepsilon_{3} \sum_{i=1}^{3} \sum_{j=1}^{3} \sum_{k=1}^{3} \epsilon_{i j k} \widetilde{L}_{i} \chi_{j} \rho_{k}\right. \\
& +k_{1} \epsilon_{i j k} \rho_{i} \chi_{j} \eta_{k}+k_{1}^{\prime} \epsilon_{i j k} \rho_{i}^{\prime} \chi_{j}^{\prime} \eta_{k}^{\prime}+\sum_{i=1}^{3} \widetilde{Q}_{1}\left(\zeta_{1 i} \eta^{\prime} \tilde{u}_{i}^{c}+\zeta_{2 i} \rho^{\prime} \widetilde{d}_{i}^{c}\right)+\sum_{\alpha=2}^{3} \widetilde{Q}_{\alpha}\left(\sum_{i=1}^{3} \omega_{1 \alpha i} \eta \widetilde{d}_{i}^{c}+\omega_{2 \alpha i} \rho \tilde{u}_{i}^{c}\right) \\
& \left.+\sum_{i=1}^{3} \sum_{j=1}^{3} \sum_{k=1}^{3} s_{1 i j k} \widetilde{d}_{i}^{c} \widetilde{d}_{j}^{c} \tilde{u}_{k}^{c}+\text { H.c. }\right] .
\end{aligned}
$$

\section{APPENDIX B: NUMERICAL ANALYSIS OF MASS MATRICES}

Here we show explicitly the numerical values of each entry of the mass matrices in Eqs. (17) and (35) using the parameters given in Eq. (24) and $m^{\prime}=-3780.4159 \mathrm{GeV}$. For the charged sector we have

$$
X=\left(\begin{array}{ccccccccc}
0.0 & -0.005 & -0.005 & 0.0 & 0.0 & 0.0 & 0.0 & -0.024 & 0.0 \\
0.005 & 0.0 & -1.851 & 0.0 & 0.0 & 0.0 & 0.0 & -235.702 & 0.0 \\
0.005 & 1.851 & 0.0 & 0.0 & 0.0 & 0.0 & 0.0 & -235.702 & 0.0 \\
0.0 & 0.0 & 0.0 & 3000.0 & 0.0 & -0.462 & 0.0 & 80.071 & 0.0 \\
0.0 & 0.0 & 0.0 & 0.0 & 3000.0 & 0.0 & 9.237 & 0.0 & -923.707 \\
0.0 & 0.0 & 0.0 & 9.237 & 0.0 & -150.0 & 0.0 & 59.868 & 0.0 \\
0.0 & 0.0 & 0.0 & 0.0 & -0.462 & 0.0 & -150.0 & 0.0 & -0.236 \\
0.0 & 0.0 & 0.0 & -0.462 & 0.0 & 471.405 & 0.0 & -250.0 & 0.0 \\
-0.004 & -40.864 & -40.864 & 0.0 & 461.854 & 0.0 & -10.379 & 0.0 & -350.000
\end{array}\right)
$$


and for the neutral sector

$Y^{0}=\left(\begin{array}{cccccccccccc}0.0 & 0.0 & 0.0 & 0.0 & 0.0 & 0.0 & 0.0 & 0.0 & 0.024 & 0.0 & 0.004 & 0.0 \\ 0.0 & 0.0 & 0.0 & 0.0 & 0.0 & 0.0 & 0.0 & 0.0 & 235.702 & 0.0 & 40.864 & 0.0 \\ 0.0 & 0.0 & 0.0 & 0.0 & 0.0 & 0.0 & 0.0 & 0.0 & 235.702 & 0.0 & 40.864 & 0.0 \\ 0.0 & 0.0 & 0.0 & 3000.0 & 0.0 & 0.0 & 6.532 & -0.327 & -56.619 & 0.327 & 0.0 & 0.0 \\ 0.0 & 0.0 & 0.0 & 0.0 & 3000.0 & 0.0 & 3.771 & -0.189 & 32.689 & -0.189 & -377.102 & 754.204 \\ 0.0 & 0.0 & 0.0 & 0.0 & 0.0 & -3780.416 & 0.0 & 0.0 & 99.391 & -0.573 & -573.290 & 1146.580 \\ 0.0 & 0.0 & 0.0 & 6.532 & 3.771 & 0.0 & 0.0 & -150.0 & -59.868 & 0.0 & 10.379 & 0.0 \\ 0.0 & 0.0 & 0.0 & -0.327 & -0.189 & 0.0 & -150.0 & 0.0 & 0.0 & -471.405 & 0.0 & 0.236 \\ 0.024 & 235.702 & 235.702 & -56.619 & 32.689 & 99.391 & -59.868 & 0.0 & 0.0 & -250.0 & -1.197 & 0.0 \\ 0.0 & 0.0 & 0.0 & 0.327 & -0.189 & -0.573 & 0.0 & -471.405 & -250.0 & 0.0 & 0.0 & -0.236 \\ 0.004 & 40.864 & 40.864 & 0.0 & -377.102 & -573.290 & 10.379 & 0.0 & -1.197 & 0.0 & 0.0 & -350.0 \\ 0.0 & 0.0 & 0.0 & 0.0 & 754.204 & 1146.580 & 0.0 & 0.236 & 0.0 & -0.236 & -350.0 & 0.0\end{array}\right.$

Here we show that the relevant parameters for the leptons masses are $\lambda_{2,3}$. We note that there are four types of parameters in the mass matrices in Eqs. (24). First we have the dimensionless Yukawa couplings in the usual leptons $\lambda_{2,3}$ and in the supersymmetric partners $f_{1}, f_{1}^{\prime}$. We also have the mass dimension parameters $\mu_{0 a}$ and $\mu_{\eta, \rho, \chi}$ and $m_{\lambda}$ and $m^{\prime}$ which are soft terms in Eq. (A4). Of all these parameters we expect that the relevant ones in the charged lepton and neutrinos are $\lambda_{2,3}$. To show this we consider several choices of the parameters as follows (below all masses are in $\mathrm{GeV}$ ).

Case 1:

$$
\begin{gathered}
\lambda_{2 e \mu}=0.0, \lambda_{2 e \tau}=0.0, \lambda_{2 \mu \tau}=0.0, \\
\lambda_{3 e}=0.0, \lambda_{3 \mu}=0.0, \lambda_{3 \tau}=0.0 \\
\left.\mu_{0 e}=\mu_{0 \mu}=0.0 ; \mu_{0 \tau}=0.0 \quad \text { (in } \mathrm{GeV}\right) .
\end{gathered}
$$

Charged sector masses:

3186.03,3001.10,557.17,196.55,149.30,16.85,0,0,0.

Neutral sector masses:

$$
\begin{aligned}
& -4162.22,3260.47,3001.10,557.79,-557.17,450.14, \\
& -330.68,17.18,-17.01,0,0,0 .
\end{aligned}
$$

Case 2:

$$
\begin{aligned}
\lambda_{2 e \mu} & =0.0, \lambda_{2 e \tau}=0.0, \lambda_{2 \mu \tau}=0.0, \\
\lambda_{3 e} & =0.0, \lambda_{3 \mu}=0.0, \lambda_{3 \tau}=0.0, \\
\mu_{0 e} & =\mu_{0 \mu}=0.0 ; \mu_{0 \tau}=2 \times 10^{-8} \quad(\text { in } \mathrm{GeV}) .
\end{aligned}
$$

Charged sector masses:

$$
\begin{aligned}
& 3186.03,3001.10,557.17,196.55,149.30,16.85,1.92 \\
& \times 10^{-12}, 0,0
\end{aligned}
$$

Neutral sector masses:

$$
\begin{aligned}
& -4162.22,3260.47,3001.10,557.79,-557.17,450.14, \\
& -330.68,17.18,-17.01,2.80 \times 10^{-21}, 0,0 .
\end{aligned}
$$

Case 3:

$$
\begin{gathered}
\lambda_{2 e \mu}=0.0, \lambda_{2 e \tau}=0.0, \lambda_{2 \mu \tau}=0.0, \\
\lambda_{3 e}=0.0001, \lambda_{3 \mu}=1.0, \lambda_{3 \tau}=1.0, \\
\left.\mu_{0 e}=\mu_{0 \mu}=0.0 ; \mu_{0 \tau}=0.0 \quad \text { (in } \mathrm{GeV}\right) .
\end{gathered}
$$

Charged sector masses:

$$
\text { 3186.05,3001.11,584.85,282.30,149.41,204.55,2.10 }
$$$$
\times 10^{-10}, 0,0 \text {. }
$$

Neutral sector masses:

$$
\begin{aligned}
& -4162.22,3260.47,3001.10,585.18,-585.18,453.22, \\
& -344.14,283.14,-271.99,1.23 \times 10^{-11}, 0,0 .
\end{aligned}
$$

Case 4:

$$
\begin{aligned}
& \lambda_{2 e \mu}=0.001, \lambda_{2 e \tau}=0.001, \lambda_{2 \mu \tau}=0.393, \\
& \lambda_{3 e}=0.0, \lambda_{3 \mu}=0.0, \lambda_{3 \tau}=0.0, \\
& \mu_{0 e}=\mu_{0 \mu}=0.0 ; \mu_{0 \tau}=0.0 \quad(\text { in } \mathrm{GeV}) .
\end{aligned}
$$

Charged sector masses:

$3186.03,3001.10,557.17,196.55,149.30,16.85,1.85,1.85,0$.

Neutral sector masses:

$$
\begin{aligned}
& -4162.22,3260.47,3001.10,557.79,-557.17,450.14, \\
& -330.68,17.18,-17.01,0,0,0 .
\end{aligned}
$$


Case 5:

$$
\begin{aligned}
\lambda_{2 e \mu} & =0.001, \lambda_{2 e \tau}=0.001, \lambda_{2 \mu \tau}=0.393, \\
\lambda_{3 e} & =0.0001, \lambda_{3 \mu}=1.0, \lambda_{3 \tau}=1.0, \\
\mu_{0 e} & =\mu_{0 \mu}=0.0 ; \mu_{0 \tau}=0.0 \quad(\text { in } \mathrm{GeV}) .
\end{aligned}
$$

Charged sector masses:

3186.03,3001.11,584.85,282.30,204.55,149.41,1.78,0.105,0.
Neutral sector masses:

$$
\begin{aligned}
& -4162.22,3260.47,3001.10,585.19,-585.19,453.22, \\
& -344.14,283.14,-271.99,1.23 \times 10^{-11}, 0,0 .
\end{aligned}
$$

Notice that the values of the masses in the charged sector are not significantly affected by the values of $\mu_{0 a}$.
[1] SuperKamiokande Collaboration, Y. Fukuda et al., Phys. Rev. Lett. 81, 1562 (1998); 82, 2644 (1999).

[2] Homestake Collaboration, B.T. Cleveland et al., Astrophys. J. 496, 505 (1998); Kamiokande Collaboration, Y. Fukuda et al., Phys. Rev. Lett. 77, 1683 (1996); GALLEX Collaboration, W. Hampel et al., Phys. Lett. B 477, 127 (1999); SAGE Collaboration, J.N. Abdurashitov et al., Phys. Rev. Lett. 77, 4708 (1996); Phys. Rev. C 60, 055801 (1999); SNO Collaboration, Q.R. Ahmad et al., Phys. Rev. Lett. 87, 071301 (2001).

[3] LSND Collaboration, C. Athanassopoulos et al., Phys. Rev. Lett. 77, 3082 (1996); 81, 1774 (1998).

[4] F. Pisano and V. Pleitez, Phys. Rev. D 46, 410 (1992); P.H. Frampton, Phys. Rev. Lett. 69, 2889 (1992); R. Foot, O.F. Hernandez, F. Pisano, and V. Pleitez, Phys. Rev. D 47, 4158 (1993).

[5] U. Okamoto and M. Yasue, Phys. Lett. B 466, 267 (1999); T. Kitabayashi and M. Yasue, Phys. Rev. D 63, 095002 (2001); Phys. Lett. B 508, 85 (2001); Nucl. Phys. B609, 61 (2001); Phys. Rev. D 63, 095006 (2001); J.C. Montero, C.A. de S. Pires, and V. Pleitez, Phys. Lett. B 502, 167 (2001); hep-ph/0112203.

[6] H.E. Haber and G.L. Kane, Phys. Rep. 117, 75 (1985).

[7] L.J. Hall and M. Suzuki, Nucl. Phys. B231, 419 (1984).

[8] T. Banks, Y. Grossman, E. Nardi, and Y. Nir, Phys. Rev. D 52, 5319 (1995).

[9] M.A. Diaz, J.C. Romão, and J.W.F. Valle, Nucl. Phys. B524, 23 (1998)
[10] F. Borzumati and Y. Nomura, Phys. Rev. D 64, 053005 (2001); F. Borzumati, K. Hamaguchi, and T. Yanagida, Phys. Lett. B 497, 259 (2001); F. Borzumati, K. Hamaguchi, Y. Nomura, and T. Yanagida, hep-ph/0012118.

[11] R.N. Mohapatra, Phys. Rev. D 34, 3457 (1986).

[12] J.C. Romão and J.W.F. Valle, Nucl. Phys. B381, 87 (1992).

[13] S. Davison and M. Losada, Phys. Rev. D 65, 075025 (2002).

[14] T.V. Duong and E. Ma, Phys. Lett. B 316, 307 (1993).

[15] J.C. Montero, C.A. de S. Pires, and V. Pleitez, Phys. Rev. D (to be published), hep-ph/0103096.

[16] J.C. Montero, V. Pleitez, and M.C. Rodriguez, Phys. Rev. D 65, 035006 (2002).

[17] M. Capdequi-Peyranere and M.C. Rodriguez, Phys. Rev. D 65, 035001 (2002).

[18] H.N. Long and P.B. Pal, Mod. Phys. Lett. A 13, 2355 (1998).

[19] P. Das, P. Jain, and D.W. Mckay, Phys. Rev. D 59, 055011 (1999).

[20] Particle Data Group, D.E. Groom et al., Eur. Phys. J. C 15, 1 (2000).

[21] Z. Maki, M. Nakagawa, and S. Sakata, Prog. Theor. Phys. 8, 246 (1962).

[22] K.S. Babu and E. Ma, Phys. Rev. Lett. 61, 674 (1988).

[23] J. C. Montero, C. A. de S. Pires, and V. Pleitez, Phys. Rev. D 65, 095001 (2002).

[24] R. Foot, H. Lew, X.-G. He, and G.C. Joshi, Z. Phys. C 44, (1989); E. Ma, Phys. Rev. Lett. 81, 1171 (1998).

[25] H.E. Haber, Nucl. Phys. B (Proc. Suppl.) 101, 217 (2001). 